\title{
The Remaining Useful Life Estimation of Lithium-ion Batteries Based on the HKA -ML-ELM Algorithm
}

\author{
Yanying $\mathrm{Ma}^{1,2}$, Dongxu Shen ${ }^{1,2}$, Lifeng $\mathrm{Wu}^{1,2}$, Yong Guan ${ }^{1,2}$, Hong Zhu ${ }^{1,2, *}$ \\ ${ }^{1}$ College of Information Engineering, Capital Normal University, Beijing 100048, China; \\ ${ }^{2}$ Beijing Key Laboratory of Electronic System Reliability Technology, Capital Normal University, \\ Beijing 100048, China \\ *E-mail: zhuh_coie@sina.com
}

doi: $10.20964 / 2019.08 .44$

Received: 8 April 2019 / Accepted: 4 June 2019 / Published: 30 June 2019

\begin{abstract}
Lithium-ion batteries have become the core energy supply component for many electronic devices. An accurate prediction of the remaining useful life (RUL) of lithium-ion batteries is of great significance for battery management and ensuring the reliability of electronic devices. The extreme learning machine (ELM) algorithm has been applied to predict the RUL of lithium-ion batteries; however, there are some disadvantages in this method: (i). the single hidden layer structure of the ELM necessarily restricts its ability to capture effective features in high-dimensional data. (ii). the input weights and biases of the ELM are generated randomly, which affects its prediction accuracy. To overcome these problems, this paper proposes an HKA-ML-ELM method for predicting the RUL of lithium-ion batteries. First, a new multi-layer ELM (ML-ELM) network is constructed. By adding an input layer into the last individual ELM of the ML-ELM and implementing the random selection of these input nodes to partially connect with the hidden layer, the network has higher robustness and can effectively prevent over-fitting. Second, the heuristic Kalman algorithm (HKA) is used to optimize the input weights and biases parameters of the ML-ELM, which improves the prediction accuracy. Finally, RUL prediction experiments are carried out for battery packs with different rated capacities and different discharge currents. The experimental results verify the effectiveness of the proposed method. The comparisons with other algorithms show that the proposed method has better prediction accuracy.
\end{abstract}

Keywords: Lithium-ion battery, RUL prediction, ML-ELM, HKA

\section{FULL TEXT}

(C) 2019 The Authors. Published by ESG (www.electrochemsci.org). This article is an open access article distributed under the terms and conditions of the Creative Commons Attribution license (http://creativecommons.org/licenses/by/4.0/). 